\title{
Anti-tumor effect of trametinib in bladder cancer organoid and the underlying mechanism
}

\author{
Mohamed Elbadawy ${ }^{1}$, Yomogi Sato ${ }^{1}$, Takashi Mori ${ }^{2}$, Yuta Goto ${ }^{1}$, Kimika Hayashi ${ }^{1}$, \\ Megumi Yamanaka ${ }^{1}$, Daigo Azakami ${ }^{1}$, Tsuyoshi Uchide ${ }^{1}$, Ryuji Fukushima ${ }^{1}$, Toshinori \\ Yoshida $^{1}$, Makoto Shibutani ${ }^{1}$, Mio Kobayashi ${ }^{1}$, Yuta Shinohara ${ }^{1}$, Amira Abugomaa ${ }^{1}$, \\ Masahiro Kaneda ${ }^{1}$, Hideyuki Yamawaki ${ }^{3}$, Tatsuya Usui ${ }^{1}$, and Kazuaki Sasaki ${ }^{1}$ \\ ${ }^{1}$ Tokyo University of Agriculture and Technology \\ ${ }^{2}$ Gifu University \\ ${ }^{3}$ Kitasato University
}

October 20, 2020

\begin{abstract}
Bladder cancer (BC), a main neoplasm of urinary tract, is usually inoperable and unresponsive to chemotherapy, indicating a necessity for more effective therapies. As a novel experimental model for muscle-invasive BC, we previously established a culture method of $\operatorname{dog} \mathrm{BC}$ organoids. In the present study, the detailed in vitro and in vivo anti-tumor effects of trametinib were investigated by using this model. In each BC organoid strain, epidermal growth factor receptor (EGFR)/ERK signaling was upregulated compared with normal bladder cells. Trametinib even at a low concentration inhibited the cell viability of $\mathrm{BC}$ organoids and the activation of ERK through decreasing expression of c-Myc, ELK1, SIK1, and PLA2G4A. Trametinib arrested cell cycle of BC with few apoptoses. Additionally, trametinib decreased expression of CD44, while YAP1 was unexpectedly upregulated. Dual treatment of BC organoids with trametinib and YAP inhibitor, verteporfin extremely inhibited the cell viability with apoptosis induction. Moreover, trametinib induced basal to luminal transformation of BC organoids by upregulating luminal markers (ERBB2 and GATA3) and downregulating basal ones (CK5 and DSG3). In vivo, trametinib decreased the tumor growth of $\mathrm{BC}$ organoids in mice and the xenograft-derived organoids from trametinib-administered mice showed enhanced sensitivity to carboplatin due to MSH2 upregulation. Our data suggested a new strategy of trametinib-YAP inhibitor or trametinib-carboplatin combination as a promising treatment of BC. Dog BC organoid model may hopefully become a promising research tool for human muscle-invasive BC in near future.
\end{abstract}

\section{Introduction}

Bladder cancer (BC), a carcinoma of bladder, is the most common and complex neoplasm of the urinary tract and is associated with high morbidity and mortality (Kamat, et al. 2016). The BC in human patients is mostly non-muscle invasive (NMI) type with a positive prognosis, while to a lesser degree it is muscleinvasive (MI) with a worse prognosis. The MIBC is mainly of basal type, high intricacy, stemness, epithelialmesenchymal transition (EMT), often metastatic, and difficult to treat (Rentsch, et al. 2017). Contrarily, the NMIBC is mainly of luminal papillary type and less complex than MIBC (Kamat, et al. 2016). Although it is the fifth most common neoplasm in the US, the precise experimental culture models that mimic the biology of the disease are few.

In dogs, $\mathrm{BC}$ constitutes about $2 \%$ of all naturally occurring cancers, a similar rate to that found in humans (Lerner, et al. 2006). Besides, it mostly resembles human MIBC in histopathology and gene profiles and then could be a valuable research model for this disease (Elbadawy, et al. 2019). In the previous study, we established a novel experimental organoid model of MIBC from BC diseased dogs using their urine 
samples (Elbadawy, et al. 2019). In this model, the MIBC characteristics were successfully recapitulated. Interestingly, this model could detect the difference of drug sensitivity for each patient to the sole and/or combined anti-cancer therapies.

The mitogen-activated protein kinase (MAPK) pathway, in which mitogen-activated extracellular signalregulated kinase (MEK) enzyme is incorporated, is constitutively activated in many tumors where it promotes cell differentiation, proliferation, angiogenesis, and survival (Sebolt-Leopold 2000). The activation of MEK1/2, in turn, phosphorylates ERK1/2, which activates multiple substrates and transcription factors (Roberts and Der 2007). The overexpression and/or mutation of growth factor receptors such as epidermal growth factor (EGF) receptor (EGFR), erbB2 receptor, and others have also been observed in many cancers (Porter and Vaillancourt 1998). Therefore, MEK has emerged as a promising anti-cancer therapeutic target (Solit, et al. 2006).

Trametinib is a type III allosteric non-competitive and highly selective MEK1/2 inhibitor (Gilmartin, et al. 2011). It suppressed RAF-dependent MEK phosphorylation and extended the inhibition of phosphorylated ERK (Gilmartin, et al. 2011). Trametinib has been approved by The United States Food and Drug Administration for treatment of several cancers that harbor BRAF V600E mutation including metastatic non-small cell lung cancer (Kelly 2018), metastatic melanoma (combined with dabrafenib) (Hoffner and Benchich 2018), and locally advanced or metastatic anaplastic thyroid cancer (Subbiah, et al. 2018). Nevertheless, previous reports concerning the use of trametinib in BC therapy were scarce and not well investigated. Since our established dog BC organoid model reflected the most features of human MIBC, the current study was carried out to investigate the effects of trametinib on $\mathrm{BC}$ organoids.

\section{Methods}

\section{Ethics approval}

Collection of urine samples, generation of bladder cancer (BC) organoids, and experiments with organoids were carried out under the direction of the Institute Animal Care and Use Committee of Tokyo University of Agriculture and Technology and approved by the ethical committee (Approval number: 0016012). Written informed consent for the present study was obtained from all dog owners. In total, five strains of BC organoids were used. Dog clinical information on each dog is listed in Table 1.

\section{Anti-cancer drugs and antibodies}

Anti-cancer drugs used in the present study were as follows: trametinib (Cayman Chemical, Ann Arbor, USA); carboplatin (FUJIFILM WAKO Pure Chemical Corporation, Osaka, Japan); yes-associated protein (YAP)1 inhibitor, verteporfin (VP) (Sigma-Aldrich, St. Louis, USA), mitoxantrone; vinblastine (Cayman). The antibodies used were as follows: total EGFR; phosphor-EGFR; phospho-ERK; total-ERK; total-YAP1 (Cell Signaling Technology, Inc., Danvers, MA, USA); CK5; total-VCP (GeneTex, Inc., Irvine, CA, USA); total-cyclin D1; MSH2 (Bioss, Inc., Woburn, MA, USA); total-CD44 (Bethyl Laboratories, Montgomery, TX, USA). Fluorescent secondary antibodies used were as follows: Alexa Fluor 488 goat anti-rabbit IgG; Alexa Fluor 488 goat anti-mouse IgG; (Thermo Fisher Scientific Inc. Waltham, MA, USA). Horseradish peroxidase (HRP)-conjugated anti-rabbit IgG (Cayman); and HRP-conjugated anti-mouse IgG (Millipore, Temecula, CA, USA).

\section{Organoid Culture}

Culture medium, supplements, conditions, handlings, and passages of the BC organoids were described before in our previous studies (Elbadawy, et al. 2019). Briefly, the culture medium was composed of Advanced DMEM with 50\% Wnt, Noggin, and R-spondin conditioned medium; 1\% GlutaMax; $100 \mathrm{ug} \mathrm{mL}^{-1}$ Primocin (Thermo Fisher Scientific); $1 \mathrm{mM}$ N-Acetyl-L-cysteine; $10 \mathrm{mM}$ nicotinamide (Sigma-Aldrich); $50 \mathrm{ng} \mathrm{\textrm {mL } ^ { - 1 }}$ mouse EGF (PeproTech, Rocky Hill, NJ, USA); and 500 nM A83-01(Adooq Bioscience, Irvine, CA, USA).

\section{Western blotting}


The immuno-related procedures used comply with the recommendations made by the British Journal of Pharmacology (Alexander et al., 2018). To check the expression and activation level of EGF signal-related proteins, $1 \times 10^{5}$ cells of BC organoid were embedded in $40 \mu \mathrm{L}$ Matrigel with culture media in a 24 -well culture plate and incubated. Subsequently, cells were harvested and protein lysates were obtained using RIPA lysis buffer (Sigma-Aldrich) supplemented with 1\% protease inhibitor cocktail (Sigma-Aldrich). Immunoblotting was carried out according to standard procedures. An equal amount of proteins $(10 \mu \mathrm{g})$ was loaded and separated by SDS-PAGE (7.5\%) and transferred to nitrocellulose membrane (Wako). After blocking, the membranes were incubated with primary antibody (total-EGFR; 1:500, phospho-EGFR; 1:200, total-ERK; 1:500; phospho-ERK; 1:500; total-VCP; 1:500) at $4^{\circ} \mathrm{C}$ overnight followed by secondary antibody (1:500 for 1 h) of goat anti-rabbit and ECL Prime (GE Healthcare, Pittsburgh, PA, USA). Images were captured with a LAS-3000 image analyzer (Fuji Film, Tokyo, Japan) and quantified by ImageJ software.

\section{Histology (H\&E staining)}

Sections prepared from trametinib- or vehicle-treated BC organoids or their xenograft-derived tumors were prepared and stained with hematoxylin and eosin (H\&E) as described before (Elbadawy, et al. 2019). Briefly, the organoids or tumor tissues were fixed at room temperature in $4 \%$ paraformaldehyde (PFA)/ phosphate-buffered saline (PBS) for 2-3 h and then embedded in paraffin. Preparation of sections and staining procedures were performed according to the standard procedures. Images were captured by using a light microscope (BX-52). Quantification of basal to luminal transformation of organoids was carried out using ImageJ software (National Institutes of Health).

\section{Cell viability assays of BC organoids}

Cell viability assay of BC organoids was carried out as described before (Usui, et al. 2017) (Elbadawy, et al. 2019) (Abugomaa, et al. 2020a). The appropriate trametinib, VP, mitoxantrone, vinblastine, and carboplatin concentrations were determined based on their blood therapeutic level in the clinic and its pharmacokinetic data (Gilmartin, et al. 2011) (Dong, et al. 2018) (Gaver, et al. 1988). Concisely, 2x10 cells of each $\mathrm{BC}$ organoid were embedded in $10 \mu \mathrm{l}$ Matrigel with culture media in a 96-well culture plate and incubated for $24 \mathrm{~h}$. Later, cells were treated with DMSO or each drug (trametinib: 0.03, 0.1, 0.3 and $1 \mu \mathrm{M}$ ), VP: $0.3 \mu \mathrm{M}$, carboplatin: $0.1,1,10$ and $100 \mu \mathrm{g} \mathrm{ml}{ }^{-1}$, mitoxantrone: $0.1,1,10$ and $100 \mu \mathrm{g} \mathrm{ml} l^{-1}$ or vinblastine: $0.1,1,10$ and $100 \mu \mathrm{g} \mathrm{ml}^{-1}$ ) for $72 \mathrm{~h}$. Cell viability was examined using PrestoBlue kit (Thermo Fisher Scientific Inc.) by a microplate reader( TECAN, Seestrasse, Switzerland) at an emission wavelength of $585 \mathrm{~nm}$. Bright-field images were captured using the light microscope (BX-52; Olympus) and the readings of the microplate reader were graphed using Sigma Plot software (Systat Software, Inc. San Jose, CA. USA).

\section{Cell cycle analysis by flow cytometry}

Cell cycle analysis was carried out to identify cell populations in various phases of the cell cycle after trametinib treatment. For this purpose, BC organoid cells $\left(2 \times 10^{5}\right.$ per well) were seeded in 24 -well plates. The organoid cells were treated with DMSO or trametinib for $24 \mathrm{~h}$. Thereafter, the cells were harvested, trypsinized, washed by PBS, fixed with $70 \%$ ethanol, and kept at $-20^{\circ} \mathrm{C}$ for $20 \mathrm{~min}$. Subsequently, the cells were treated with Rnase $\left(200 \mu \mathrm{g} \mathrm{m}^{-1}\right.$, Sigma-Aldrich) for $30 \mathrm{~min}$ at room temperature and stained with propidium iodide $\left(50 \mu \mathrm{g} \mathrm{ml}^{-1}\right.$, Sigma-Aldrich) for $30 \mathrm{~min}$ at room temperature in the dark. The cells were then gently dissociated and passed through a $200 \mu \mathrm{m}$ nylon mesh before data acquisition using flow cytometry (Guava easyCyte, Millipore) and the phase distributions were quantified using the attached software.

\section{Quantitative real-time PCR}

Total RNA was extracted from control and trametinib-treated organoid samples using the NucleoSpin kit (Takara Bio Inc., Shiga, Japan) following the manufacturer's instructions. First-strand cDNA was prepared according to the QuantiTect Reverse Transcription Kit (QIAGEN, Hilden, Germany) instructions. Quantitative real-time PCR was carried out using a QuantiTect SYBR I Kit (QIAGEN) and a StepOnePlus Real-Time PCR System (Applied Biosystems, Waltham, MA, USA). The $\Delta \Delta$ Cq method was applied for 
quantification. The analysis was using GAPDH as a control gene. Specific dog primers used for downstream genes of ERK signaling; c-Myc, ETS transcription factor, ETS Like-1 protein (ELK1), Salt-Inducible Kinase 1 (SIK1), and phospholipase A2 group 4A(PLA2G4A); cell cycle-related genes; cyclin D1, cyclin A2, cyclin E1, CDK4, CDK6, p21, and p16; stemness markers; CD44 and YAP1; basal cell markers, CK5 and DSG3; luminal cell markers, ERBB2 and GATA3; carboplatin sensitivity-related genes; MutS Homolog (MSH)2, MutL homolog (MLH)1, and excision repair cross-complementing rodent repair deficiency, complementation group ( ERCC) 1 are listed in Table 2.

\section{Immunofluorescence staining}

Immunofluorescence staining of BC organoids and their xenograft sections were performed as described previously (Usui, et al. 2017) (Elbadawy, et al. 2019). After fixation of sections in 4\% PFA for $1 \mathrm{~h}$ and dehydration with $30 \%$ sucrose solution at $4^{\circ} \mathrm{C}$ overnight, they were embedded in OCT compound. The frozen sections were made using Leica CM3050 S Research Cryostat (Leica Biosystem, Buffalo Grove, IL, USA). Sections were blocked with 1.5\% normal goat serum (NGS)/PBS at room temperature for $1 \mathrm{~h}$. Subsequently, they were incubated with primary antibodies (cyclin D1 1:200, CD44; 1:200, YAP1; 1:200, CK5; 1:200, MSH2; 1:200) and kept at $4^{\circ} \mathrm{C}$ overnight. Thereafter, the sections were washed 3 times with PBS and incubated with secondary antibody for $1 \mathrm{~h}$. The images were captured by a confocal microscope (LSM 800; ZEISS, Copenhagen, Germany).

\section{TUNEL staining}

Detection of apoptosis in the frozen sections of BC organoids and their xenograft-derived tumor tissues was performed using the DeadEnd Fluorometric terminal deoxynucleotidyl transferase dUTP nick end labeling (TUNEL) system (Promega Co., Madison, USA) following the manufacturer's instructions. BC organoid cells $\left(1 \times 10^{5}\right.$ cells per well) were seeded and cultured for $24 \mathrm{~h}$ and then treated with trametinib $(1 \mu \mathrm{M})$ or DMSO for $72 \mathrm{~h}$. Frozen sections were prepared from treated cells and kept at $-80{ }^{\circ} \mathrm{C}$ until apoptosis assay. The subsequent procedures for apoptosis assay were carried out following the manufacturer's protocol. Concisely, after washing the slides with PBS for $5 \mathrm{~min}$, they were fixed in $4 \%$ PFA for $15 \mathrm{~min}$. Sections were then permeabilized with $2 \%$ proteinase $\mathrm{K}$ solution in PBS for 15 min. After PBS washing, sections were fixed again in $4 \% \mathrm{PFA}$ for $5 \mathrm{~min}$. The DNase I (0.5 U)-treated slide was prepared as a positive control. All sections were then treated with $100 \mathrm{ul}$ of equilibration buffer for $10 \mathrm{~min}$. After that section was treated with $100 \mathrm{ul}$ of $\mathrm{rTdT}$ incubation buffer, covered by plastic coverslips, and incubated at $37^{\circ} \mathrm{C}$ inside a humidified chamber for $60 \mathrm{~min}$, and the tailing reaction was then terminated by immersion of slides in 2X SCC solution for 15 min. Cell nuclei were counterstained with $3 \mu \mathrm{g} \mathrm{mL} \mathrm{m}^{-1}$ Hoechst (in PBS) for $5 \mathrm{~min}$. The sections were sealed, and the fluorescein12-dUTP-labeled DNA was visualized by fluorescence imaging. The images were captured by a confocal microscope (LSM 800; ZEISS).

\section{Organoid xenograft studies}

All experimental procedures were carried out following the recommendations of the 'Guide for the Care and Use of Laboratory Animals' and approved by ethics committees of Tokyo University of Agriculture and Technology (Approval number: 29-92) and the recommendations made by the British Journal of Pharmacology. To study the in vivo anti-cancer effects of trametinib against xenograft $\mathrm{BC}$ organoids, $\mathrm{BC}$ organoid cells $\left(1 \times 10^{6}\right)$ were subcutaneously implanted into the back of male SCID (C.B-17/IcrHsd-Prkdc ${ }^{\text {scid }}$, Japan SLC) mice $(\mathrm{n}=12)$. After forming a tumor of a certain size, the mice were randomly divided into two groups and trametinib or vehicle was intraperitoneally injected $\left(1 \mathrm{mg} \mathrm{kg}^{-1}, 6\right.$ days per week) for one month. Tumor dimensions were measured every week by caliber and their volumes $(\mathrm{V})$ were estimated using $\mathrm{V}=$ $1 / 2\left(\mathrm{~L} \times \mathrm{W}^{2}\right)$, where $\mathrm{L}$ is the longest dimension (length) and $\mathrm{W}$ is the width (shortest dimension). The mean tumor volumes were recorded for each group. Six weeks post-injection of organoids, mice were anesthetized by isoflurane before cervical dislocation and tumors were isolated and weighed. The sections were prepared for H\&E, TUNEL staining, and immunofluorescence staining.

\section{Statistical analysis}


Data shown are means \pm SEM. Statistical evaluations were performed using a one-way analysis of variance (ANOVA) followed by Bonferroni's test. P-values [?]0.05 were statistically significant. The data and statistical analysis comply with the recommendations of the British Journal of Pharmacology on experimental design and analysis in pharmacology (Curtis et al., 2018).

\section{Results}

\section{Effects of trametinib on cell viability and activation of cell signaling in $\operatorname{dog} B C$ organoids}

In the previous study, we established the method of dog $\mathrm{BC}$ organoids and suggested that dog $\mathrm{BC}$ organoids become a new experimental model of human MIBC (Elbadawy, et al. 2019). To clarify the molecular mechanisms in BC organoids and identify the effective molecular targeting drugs, we analyzed the activated signaling, histological characteristics, and drug sensitivity in BC organoids (Fig. 1A). Since activation of EGF-regulated signaling was reported in several human MIBC (Takeuchi, et al. 2012), we checked the expression and phosphorylation level of EGFR and ERK in dog BC organoids. As expected, expression and phosphorylation levels of EGFR and ERK in five strains of BC organoids were upregulated compared with normal bladder tissue cells (Fig. 1B and Fig. S1). Staining of organoids with H\&E showed the basal solid-like structure with squamous differentiation (Fig. 1C), which corresponded to the typical histology of MIBC in human. We next checked the effect of a MEK inhibitor, trametinib on the cell viability of BC organoid cells. Interestingly, trametinib even at low concentration $(0.03 \mu \mathrm{M})$ drastically inhibited the viability of organoid cells in all strains (Fig. 1D and E). Thereafter, we checked the time-course effects of trametinib on activation of ERK and EGFR. As presented in Fig. $1 \mathrm{~F}$ and G, trametinib completely inhibited phosphorylation of ERK in $\mathrm{BC}$ organoids even at $3 \mathrm{~h}$ after treatment and the effect continues to $24 \mathrm{~h}$. However, there was no effect on phosphorylation of EGFR. We further checked the downstream signals of ERK cascade. Among the several transcriptional genes, trametinib significantly inhibited expression of c-Myc, ELK1, SIK1, and PLA2G4A (Fig. 1H and Fig. S2). These findings indicate the potential role of ERK downstream signals in the growth and proliferation of $\mathrm{BC}$ and trametinib may become a new therapeutic agent against human MIBC.

Effects of trametinib on cell cycle arrest and apoptosis in BC organoids

We next checked whether trametinib affects cell cycle in BC organoids. In the trametinib treatment of $\mathrm{BC}$ organoid cells, the ratio of G0/G1 phase fractions was significantly elevated, whereas the G2/M phase fractions was significantly declined (Fig. 2A). To investigate the detailed mechanisms of cell arrest by trametinib in $\mathrm{BC}$ organoids, we examined the effects of trametinib on expression of cell cycle-related genes. After $24 \mathrm{~h}$ trametinib treatment, mRNA expression level of cell cycle-related genes was investigated. Among the several genes, cyclin D1, cyclin A2, cyclin E1, and CDK4 were significantly downregulated, whereas CDK6, p21, and p16 did not change (Fig. 2B). Interestingly, cyclin D1 and cyclin E1 were downregulated in a time-dependent manner, whereas cyclin A2 and CDK4 were downregulated only after $24 \mathrm{~h}$ treatment (Fig. S3). Further confirmation at protein expression level was carried out and the data revealed that cyclin D1 was significantly less expressed in the trametinib-treated organoids compared with the control (Fig. $2 \mathrm{C}$ and D). Regarding to apoptotic responses, trametinib treatment increased the ratio of TUNEL-positive cells, but the effect was minimal (Fig. $2 \mathrm{E}$ and F). These data indicate that trametinib decreased cell viability of BC organoids through cell arrest rather than apoptosis induction.

\section{Effects of trametinib on stemness of BC organoids}

Since cancer stem cell (CSC) markers are implicated in growth, invasion, angiogenesis, chemotherapy resistance, and metastasis of MIBC, the effect of trametinib on expression of two important markers, CD44 and YAP1 which were upregulated in most of MIBC (Ciamporcero, et al. 2016) was investigated. Treatment of $\mathrm{BC}$ organoids with trametinib $(1 \mu \mathrm{M})$ significantly decreased the expression level of CD44 mRNA at 12 and $24 \mathrm{~h}$, while YAP1 expression was significantly upregulated in a time-dependent manner (Fig. 3A). Further confirmation of these findings at the protein expression level was undertaken using immunofluorescence staining with CD44 and YAP1 antibodies (Fig. 3B). Considering these data, we hypothesized that a combination treatment of trametinib and YAP inhibitor more efficiently decreases the cell viability of BC organoids. To prove this hypothesis, the synergistic effect of YAP inhibitor, verteporfin (VP) $(0.3 \mu \mathrm{M})$ with trametinib at 
low concentration $(0.03 \mu \mathrm{M})$, on the viability of $\mathrm{BC}$ organoids was assessed. As expected, trametinib plus VP inhibited to a greater extent the cell viability of $\mathrm{BC}$ organoids and the effect was significantly higher compared with the sole treatment of each drug (Fig. 3C and D). Furthermore, after co-treatment with VP, the apoptotic level was assessed using TUNEL staining. Interestingly, VP plus trametinib-treated BC organoids increased apoptotic cells compared with trametinib alone or DMSO-treated (control) organoids (Fig. 3E and F). These results indicate that the combinational treatment of trametinib and YAP1 inhibitor is a good therapeutic strategy for the treatment of MIBC.

\section{Effects of trametinib on cell transformation of BC organoids}

After long term $(72 \mathrm{~h})$ trametinib treatment of $\mathrm{BC}$ organoids, we observed the morphology clearly changed. The basal solid-like structure was transformed into a luminal cystic and thin-like one (Fig. 4A and B). Trametinib treatment significantly decreased the ratio of basal-like organoids, while the luminal-like ones increased compared with control (Fig. 4C). To confirm the trametinib-induced cell transformation, we checked the expression of basal and luminal markers by using quantitative real-time PCR. Expression of mRNA of basal markers, CK5 and DSG3 was significantly decreased, while the expression of luminal ones, ERBB2 and GATA3 was significantly increased in the trametinib-treated BC organoids compared with control (Fig. $4 \mathrm{D}$ ). At the protein expression level, the immunofluorescence data revealed that trametinib significantly decreased the number of CK5-positive cells (Fig. $4 \mathrm{E}$ and $\mathrm{F}$ ). These results indicate that trametinib induced basal to luminal transformation of $\mathrm{BC}$ organoids.

\section{Effects of trametinib on tumor growth against xenografted BC organoids in immunodeficient mice}

To check the effects of trametinib in vivo, we performed a xenograft experiment of $\mathrm{BC}$ organoids and trametinib was administrated to mice after tumor-forming (Fig. 5A). Four weeks after administration, the tumor growth in the trametinib-administered mouse group was significantly arrested compared with the control group (Fig. 5B and C). Also, the tumor weight was significantly lower in the trametinib group compared with the control group (Fig. 5D). Histological analyses showed that trametinib induced EMTlike structural changes in the tumor tissue compared with the control tumor tissues (Fig. 5E). TUNEL staining showed few apoptotic changes in the tumor tissues of trametinib-administered mice compared with the control ones (Fig. 5F). We also observed that expression of CK5 in the tumor tissues of trametinibtreated mice was significantly decreased compared with the control ones (Fig. 5G and H), indicating that the luminal transformation also occurred in the tumor tissues. These results suggest the in vivo efficacy of trametinib in the treatment of MIBC.

\section{Effects of long-term trametinib administration to mice on drug sensitivity of xenograft-derived organoids}

To analyze whether the long-term trametinib administration to mice affects drug sensitivity of tumor tissues, tumor tissues from trametinib-treated and control mice were dissected for developing xenograft-derived organoids and were used for experiments (Fig. 6A). At day 3 after culture, the xenograft-derived organoids from trametinib-administered mice (XDO-T) showed a fewer number, smaller size, and lower growth speed compared with those of the control group (XDO-C) (Fig. 6B). After passaging, their organoid-forming efficiency soon recovered. The histological difference between XDO-C and XDO-T was not observed (Fig. $6 \mathrm{C}$ ). Interestingly, after the long term (four weeks) administration of trametinib, the organoid sensitivity to trametinib (Fig. 6D), mitoxantrone, and vinblastine (Fig. S4) did not change, while the sensitivity to carboplatin was enhanced (Fig. 6E). To clarify the molecular mechanisms of the upregulation of sensitivity to carboplatin, expression level of carboplatin sensitivity-related genes, MSH2, MLH1, and ERCC1 was examined. Among these genes, MSH2 was significantly upregulated in XDO-T compared with XDO-C (Fig. $6 \mathrm{~F}$ ). The protein expression level of MSH2 was also increased in XDO-T compared with XDO-C as well as in the corresponding tumor tissues (Fig. 6G). These results indicate that xenograft-derived tumor tissues did not develop resistance to trametinib after 4 weeks administration period. Additionally, trametinib enhanced the sensitivity of the XDO-T to carboplatin.

\section{Discussion}


In the current study, we investigated the effects of trametinib on dog $\mathrm{BC}$ organoids. The main findings are as follows: 1) trametinib inhibited to a higher extent the viability of organoids and the activation of ERK but not EGFR (Fig. 1 D-G) through inhibition of expression of c-Myc, ELK1, SIK1, and PLA2G4A (Fig. $1 \mathrm{H}$ \& Fig. S2), 2) trametinib inhibited G2/M phase fractions of the cell cycle and expression of cell cyclerelated genes (Fig. 2 A-D \& Fig. S3) with little apoptosis induction (Fig. 2E), 3) CD44 was downregulated while YAP1 was upregulated after trametinib treatment (Fig. 3 A and B), 4) YAP1 inhibitor, verteporfin showed synergism with trametinib (Fig. $3 \mathrm{C}-\mathrm{F}$ ), 4) trametinib induced basal to luminal transformation of $\mathrm{BC}$ organoids with upregulation of luminal markers (ERBB2 and GATA3) and downregulation of basal ones (CK5 and DSG3) (Fig. 4), 5) trametinib inhibited tumor growth of engrafted BC organoids in mice with basal to luminal transformation of tumor tissues (Fig. 5), 6) xenograft-derived organoids from trametinibadministered mice showed specifically enhanced sensitivity to carboplatin through upregulation of MSH2 expression (Fig. 6 E-G). Collectively (Fig. 6H), our data suggest the value of our organoid model as a new tool to provide new therapeutic insights for BC.

While $\mathrm{BC}$ is frequent, its management is often difficult especially for the MI type which is solid and of basal origin (Rentsch, et al. 2017) with more stemness and EMT (Sjodahl, et al. 2012) and is often metastatic (Choi, et al. 2014b). In recent years, BC overall incidence and mortality have shown a steady upward trend and have become a significant challenge to people's health (Antoni, et al. 2017). To improve treatment strategies, new research models are necessary, for which organoids are a promising one. Organoids are generated from auto-renovating stem cells which typically recapitulate the in vivo architecture of original tissues, functions, and genetic and molecular imprints (Elbadawy, et al. 2020b). It holds great promise for establishing new personalized treatments and analyzing drug resistance in the medical field (Usui, et al. 2016) (Usui, et al. 2018a) (Usui, et al. 2018b) (Elbadawy, et al. 2018) (Abugomaa and Elbadawy 2020) (Elbadawy, et al. 2020a). The treatment strategies for BC continue to expand and their efficacy varies depending on the type, clinical stage, and associated risk factors (Prasad, et al. 2011) with a positive shift towards the more personalized therapy. For MIBC, the current standard of care is cisplatin-based chemotherapy followed by radical cystectomy, immunotherapy, gene therapy, or targeted therapy. Targeted therapy strategy functions the agent to identify oncogenic targets as cell surface antigen, a membrane protein molecule, or gene fragment of cancerous cells, and then induces necrosis and apoptosis of tumor cells. Recently, several oncogenic targets and signaling pathways were implicated and targeted, but mostly in $\mathrm{BC}$ cell lines ( $\mathrm{Su}$, et al. 2019). For example, everolimus was used to target AKT in the mTOR pathway (Pinto-Leite, et al. 2016), lapatinib to target HER2 (ERBB2) in the RTK/MAPK signaling pathway (Sakai, et al. 2018, and erlotinib to target EGFR in EGF signaling pathway \{Tsai, 2015 \#74). Among the several targets, ERK1/2 which is activated by MEK1/2 is important signaling pathways implicated in bladder carcinogenesis in both humans (Takeuchi, et al. 2012) and dogs (Hanazono, et al. 2015).

In the present study, both total expression and phosphorylation level of EGFR and ERK were upregulated in the BC organoids (Fig. 1B, Fig. S1). Thus, the therapeutic targeting of an upstream protein of ERK, MEK is important to stop its growth (Cohen 2002). Trametinib, the promising MEK1/2 inhibitor has been approved for the treatment of several cancers (Kelly 2018), but not yet for BC due to the scarcity of data. In the present study, we investigated, in details and for the first time, the effect of trametinib on our established BC organoids of basal solid-like structure (Fig. 1C), as a model for human MIBC (Elbadawy, et al. 2019). The treatment of $\mathrm{BC}$ organoids with trametinib significantly inhibited phosphorylation of ERK (Fig. 1F and G) but not EGFR. We further checked the downstream signals of ERK cascade and found that, among the several signals, trametinib significantly inhibited the c-Myc, ETS transcription factor, ELK1, and SIK1 signals (Fig. 1, H). Interestingly, our results showed that ELK1 and SIK1 are targets of trametinib in BC. ELK1 was recently shown to be involved in BC progression (Kawahara, et al. 2015) and here for the first time in $\mathrm{BC}$ organoids progression. Also, in the present study and for the first time, SIK1 was shown to be involved in BC progression. Recently, SIK1 was reported to be upregulated and involved in progression of several cancers including colorectal cancer (Huang, et al. 2019), medulloblastoma (Huang, et al. 2020), and non-small cell lung cancer (Hollstein, et al. 2019) and its downregulation suppressed these tumors. These data imply that trametinib inhibited BC growth by targeting the ERK downstream signals, ELK1 and SIK1. 
Since many cancer stem cell (CSC) markers are implicated in growth, invasion, angiogenesis, chemotherapy resistance, and metastasis of MIBC (Abugomaa, et al. 2020b), the effect of trametinib on two important stem cell markers, CD44 and YAP1 was investigated in the BC organoids. Treatment of BC organoids with trametinib successfully inhibited CD44, but unexpectedly upregulated YAP1 (Fig. 3A and B). CD44 is a cell surface adhesion molecule located at the basal layer of the normal urothelium (Brandt, et al. 2009) and involved in cancer cell proliferation, migration, metastasis, angiogenesis, EMT, aggressiveness, and disease progression (Naor, et al. 1997). Therefore, inhibition of CD44-related stemness by trametinib decreased the viability of $\mathrm{BC}$ organoids in the present study. Of note, CD44 inhibition might be a consequence of inhibition of the ERK signaling pathway (Maehara, et al. 2017). In other cancers, trametinib treatment inhibited ERK phosphorylation and decreased CD44 expression in malignant pleural mesothelioma cells (Cho, et al. 2017) and esophageal squamous cell carcinoma (Maehara, et al. 2017). YAP1 is a downstream transcription coactivator and a major nuclear effector of the Hippo tumor suppressor pathway (Beverdam, et al. 2013). YAP1 is highly expressed in most BC patients, confers CSC traits (Johnson and Halder 2014), promotes tumorigenesis (Ooki, et al. 2018), protects BC from chemotherapy-induced DNA damage (Ciamporcero, et al. 2016), and contributes to the progression of it to an advanced stage and poor prognosis (Liu, et al. 2013). The YAP1 inhibitor, VP (a photodynamic therapy to treat macular degeneration of the retina) was used recently to target YAP1 signaling pathway in mesothelioma (Kandasamy, et al. 2020), prostate cancer (Jiang, et al. 2015), melanoma (Feng, et al. 2014), and BC cell lines (Ooki, et al. 2018). In BC, VP was successfully shown to inhibit the progression of T24 and RP-B-01 BC cell lines in a dose-dependent manner via induction of apoptosis, decreasing SOX2 expression, and enhancing chemotherapy efficacy (Ooki, et al. 2018). In the present study, VP successfully inhibited the cell viability of BC organoid cells in a dose-dependent manner (data are not shown) and showed synergetic effects on the trametinib-treated BC organoid cells through induction of apoptosis (Fig. 3E and F). These results and ours highlight the oncogenic role of the YAP1 signaling pathway in BC and its pharmacological targeting by VP is important to arrest BC progression by induction of apoptosis and lowering the stemness.

BC can be grouped into basal and luminal subtypes (Choi, et al. 2014a). Basal-subtype BC is muscleinvasive, more aggressive, metastatic, difficult to treat with poor outcomes, and higher EMT due to its enriched CSC traits than luminal one (Choi, et al. 2014a). As noted, the tested strains of BC organoids in the current study are of basal solid-like structure with squamous differentiation (Fig. 1C \& Fig. 4A and B) that mimics the human MIBC (Choi, et al. 2014a) (Elbadawy, et al. 2019). Similarly, the basal MIBC showed more squamous features (Choi, et al. 2014b) and expression of the higher molecular weight keratins (CK5, CK6, CK14) and CD44 expression (Choi, et al. 2014b) that characterizes cells in the basal layer of normal urothelium (Volkmer, et al. 2012) and MIBC with squamous differentiation (Choi, et al. 2014b). This kind of BC revealed more aggressiveness and had shorter overall survival (Hurst and Knowles 2014). Interestingly, trametinib treatment induced morphological and molecular transformation of $\mathrm{BC}$ organoids (Fig. 4). These data indicate the efficacy of trametinib in decreasing the basal and squamous features of $\mathrm{BC}$ organoids and therefore might help for decreasing the aggressiveness of MIBC.

The high invasions, metastasis, and resistance of MIBC to most of the chemotherapy agents necessitate the continual search for new combinational treatment strategies to improve the survival rate of patients, especially in precision medicine. Using chemotherapy agents alone is usually toxic and intolerant for the patients. The treatment modality that combines two or more therapeutic agents with a different mechanism of actions with lowering their doses, is a cornerstone of cancer therapy as it potentially reduces resistance and metastasis and increases survival rate (Bayat Mokhtari, et al. 2017). Therefore, new therapeutic strategies that provide effective results and target the survival pathways at an affordable cost are being considered. In the present study, XDO-T showed a significantly enhanced sensitivity to the platinum-based adjuvant therapy, carboplatin (Fig. 6E). Analyzing this valuable effect, we found that among the several genes responsible for enhancing carboplatin sensitivity, MSH2 was significantly upregulated (Fig. 6F). Recently, high MSH2 expression was shown to mediate the death of MIBC cells by the platinum-based chemotherapy (Goodspeed, et al. 2019) and predicting the response to it (Mucaki, et al. 2019). Carboplatin was tried either alone (Chun, et al. 1997) or with others like piroxicam (Allstadt, et al. 2015) or gemcitabine (Hudson 
and Lester 2010) for treatment of dog BC. However, the outcomes as median survival time and progression rate were not improved (Allstadt, et al. 2015). The combination of carboplatin with trametinib was trialed recently for the treatment of locally advanced non-small-cell lung cancer (Lin, et al. 2015), but never for human BC. Therefore, based on our findings, using trametinib with carboplatin therapy is reasonable as a new strategic option for better treatment of both human and $\operatorname{dog} \mathrm{BC}$.

In conclusion, we for the first time investigated the effect of trametinib on $\mathrm{BC}$ organoids. Our results showed the efficacy of trametinib against $\mathrm{BC}$ organoids in vitro and in vivo (Fig. $6 \mathrm{H}$ ). These data suggest that trametinib has therapeutic potential for MIBC, particularly in ERK-overexpressing ones. Further confirmation studies on the roles of ERK downstream signals, c-Myc, ELK1, SIK1, and PLA2G4A in BC organoid will help the development of future targets of MIBC.

\section{Acknowledgments}

This study was supported in part by a Grant-in-Aid for Scientific Research from the Japan Society for the Promotion of Science (JSPS). Grant Number is 20H03145.

\section{Conflict of interests}

Nothing exists.

\section{Author contributions}

M.E, Y.S., T.M., and T.U. developed the concept and methodology. M.E, Y.S., Y.G., K.H., M.Y., D.A., T.U., R.F., T.Y., M.S., M.K., Y.S., A.A., and T.U. performed the in vitro and in vivo experiments, carried out the data acquisition, analyzed the study. M.K., H.Y., T.U., and K.S. did the manuscript preparation. All authors read and approved the manuscript.

\section{Declaration of transparency and scientific rigour}

This Declaration acknowledges that this paper adheres to the principles for transparent reporting and scientific rigour of preclinical research as stated in the BJP guidelines for Design \& Analysis, Immunoblotting and Immunochemistry, and Animal Experimentation, and as recommended by funding agencies, publishers and other organizations engaged with supporting research.

\section{ORCID}

Tatsuya Usui: https://orcid.org/0000-0002-1818-2127

\section{References}

Abugomaa, A., et al.

2020a Establishment of 2.5D organoid culture model using 3D bladder cancer organoid culture. Sci Rep 10(1):9393.

Abugomaa, A., et al.

2020b Emerging Roles of Cancer Stem Cells in Bladder Cancer Progression, Tumorigenesis, and Resistance to Chemotherapy: A Potential Therapeutic Target for Bladder Cancer. Cells 9(1).

Abugomaa, Amira, and Mohamed Elbadawy

2020 Patient-derived organoid analysis of drug resistance in precision medicine: is there a value? Expert Review of Precision Medicine and Drug Development 5(1):1-5.

Allstadt, S. D., et al.

2015 Randomized phase III trial of piroxicam in combination with mitoxantrone or carboplatin for first-line treatment of urogenital tract transitional cell carcinoma in dogs. J Vet Intern Med 29(1):261-7.

Antoni, S., et al. 
2017 Bladder Cancer Incidence and Mortality: A Global Overview and Recent Trends. Eur Urol 71(1):96108.

Bayat Mokhtari, R., et al.

2017 Combination therapy in combating cancer. Oncotarget 8(23):38022-38043.

Beverdam, A., et al.

2013 Yap controls stem/progenitor cell proliferation in the mouse postnatal epidermis. J Invest Dermatol 133(6):1497-505.

Brandt, W. D., et al.

2009 Urothelial carcinoma: stem cells on the edge. Cancer Metastasis Rev 28(3-4):291-304.

Cho, H., et al.

2017 Trametinib plus 4-Methylumbelliferone Exhibits Antitumor Effects by ERK Blockade and CD44 Downregulation and Affects PD-1 and PD-L1 in Malignant Pleural Mesothelioma. J Thorac Oncol 12(3):477-490.

Choi, W., et al.

2014a Intrinsic basal and luminal subtypes of muscle-invasive bladder cancer. Nat Rev Urol 11(7):400-10.

Choi, W., et al.

2014b Identification of distinct basal and luminal subtypes of muscle-invasive bladder cancer with different sensitivities to frontline chemotherapy. Cancer Cell 25(2):152-65.

Chun, R., et al.

1997 Phase II clinical trial of carboplatin in canine transitional cell carcinoma of the urinary bladder. J Vet Intern Med 11(5):279-83.

Ciamporcero, E., et al.

2016 YAP activation protects urothelial cell carcinoma from treatment-induced DNA damage. Oncogene $35(12): 1541-53$.

Cohen, Philip

2002 Protein kinases - the major drug targets of the twenty-first century? Nature Reviews Drug Discovery $1(4): 309-315$.

Dong, L., et al.

2018 Verteporfin inhibits YAP-induced bladder cancer cell growth and invasion via Hippo signaling pathway. Int J Med Sci 15(6):645-652.

Elbadawy, M., et al.

2020a Development of Prostate Cancer Organoid Culture Models in Basic Medicine and Translational Research. Cancers (Basel) 12(4).

Elbadawy, M., et al.

2019 Establishment of a novel experimental model for muscle-invasive bladder cancer using a dog bladder cancer organoid culture. Cancer Sci 110(9):2806-2821.

Elbadawy, M., et al.

2018 Development of an Experimental Model for Analyzing Drug Resistance in Colorectal Cancer. Cancers (Basel) 10(6). 
Elbadawy, M., et al.

2020b Efficacy of primary liver organoid culture from different stages of non-alcoholic steatohepatitis (NASH) mouse model. Biomaterials 237:119823.

Feng, X., et al.

2014 Hippo-independent activation of YAP by the GNAQ uveal melanoma oncogene through a trio-regulated rho GTPase signaling circuitry. Cancer Cell 25(6):831-45.

Gaver, R. C., et al.

1988 The disposition of carboplatin in the beagle dog. Cancer Chemother Pharmacol 21(3):197-202.

Gilmartin, A. G., et al.

2011 GSK1120212 (JTP-74057) is an inhibitor of MEK activity and activation with favorable pharmacokinetic properties for sustained in vivo pathway inhibition. Clin Cancer Res 17(5):989-1000.

Goodspeed, A., A. Jean, and J. C. Costello

2019 A Whole-genome CRISPR Screen Identifies a Role of MSH2 in Cisplatin-mediated Cell Death in Muscle-invasive Bladder Cancer. Eur Urol 75(2):242-250.

Hanazono, K., et al.

2015 Epidermal growth factor receptor expression in canine transitional cell carcinoma. J Vet Med Sci 77(1):1-6.

Hoffner, B., and K. Benchich

2018 Trametinib: A Targeted Therapy in Metastatic Melanoma. J Adv Pract Oncol 9(7):741-745.

Hollstein, P. E., et al.

2019 The AMPK-Related Kinases SIK1 and SIK3 Mediate Key Tumor-Suppressive Effects of LKB1 in NSCLC. Cancer Discov 9(11):1606-1627.

Huang, C., et al.

2019 MicroRNA-17 promotes cell proliferation and migration in human colorectal cancer by downregulating SIK1. Cancer Manag Res 11:3521-3534.

Huang, S., et al.

2020 Exosomal miR-130b-3p targets SIK1 to inhibit medulloblastoma tumorigenesis. Cell Death Dis 11(6):408.

Hudson, E., and J. F. Lester

2010 Gemcitabine and carboplatin in the treatment of transitional cell carcinoma of the urothelium: a single centre experience and review of the literature. Eur J Cancer Care (Engl) 19(3):324-8.

Hurst, C. D., and M. A. Knowles

2014 Molecular subtyping of invasive bladder cancer: time to divide and rule? Cancer Cell 25(2):135-6.

Jiang, N., et al.

2015 In vivo quantitative phosphoproteomic profiling identifies novel regulators of castration-resistant prostate cancer growth. Oncogene 34(21):2764-76.

Johnson, R., and G. Halder 
2014 The two faces of Hippo: targeting the Hippo pathway for regenerative medicine and cancer treatment. Nat Rev Drug Discov 13(1):63-79.

Kamat, Ashish M., et al.

2016 Bladder cancer. The Lancet 388(10061):2796-2810.

Kandasamy, S., et al.

2020 The YAP1 Signaling Inhibitors, Verteporfin and CA3, Suppress the Mesothelioma Cancer Stem Cell Phenotype. Mol Cancer Res 18(3):343-351.

Kawahara, T., et al.

2015 Silodosin inhibits the growth of bladder cancer cells and enhances the cytotoxic activity of cisplatin via ELK1 inactivation. Am J Cancer Res 5(10):2959-68.

Kelly, R. J.

2018 Dabrafenib and trametinib for the treatment of non-small cell lung cancer. Expert Rev Anticancer Ther 18(11):1063-1068.

Lerner, Seth P, Mark Schoenberg, and Cora Sternberg

2006 Textbook of bladder cancer. Oxon, UK: Taylor and Francis.

Lin, S. H., et al.

2015 NCI 9448: Phase I study of trametinib in combination with chemoradiation for KRAS-mutant non-small cell lung cancer. Journal of Clinical Oncology 33(15):TPS7585-TPS7585.

Liu, J. Y., et al.

2013 Overexpression of YAP 1 contributes to progressive features and poor prognosis of human urothelial carcinoma of the bladder. BMC Cancer 13:349.

Maehara, O., et al.

2017 Fibroblast growth factor-2-mediated FGFR/Erk signaling supports maintenance of cancer stem-like cells in esophageal squamous cell carcinoma. Carcinogenesis 38(11):1073-1083.

Mucaki, E. J., et al.

2019 Predicting responses to platin chemotherapy agents with biochemically-inspired machine learning. Signal Transduct Target Ther 4:1.

Naor, D., R. V. Sionov, and D. Ish-Shalom

1997 CD44: structure, function, and association with the malignant process. Adv Cancer Res 71:241-319.

Ooki, A., et al.

2018 YAP1 and COX2 Coordinately Regulate Urothelial Cancer Stem-like Cells. Cancer Res 78(1):168-181.

Pinto-Leite, R., et al.

2016 mTOR inhibitors in urinary bladder cancer. Tumour Biol 37(9):11541-11551.

Porter, A. C., and R. R. Vaillancourt

1998 Tyrosine kinase receptor-activated signal transduction pathways which lead to oncogenesis. Oncogene 17(11 Reviews):1343-52.

Prasad, S. M., et al. 
2011 Urothelial carcinoma of the bladder: definition, treatment and future efforts. Nat Rev Urol 8(11):63142.

Rentsch, C. A., et al.

2017 Comprehensive Molecular Characterization of Urothelial Bladder Carcinoma: A Step Closer to Clinical Translation? Eur Urol 72(6):960-961.

Roberts, P. J., and C. J. Der

2007 Targeting the Raf-MEK-ERK mitogen-activated protein kinase cascade for the treatment of cancer. Oncogene 26(22):3291-310.

Sakai, K., et al.

2018 Anti-tumour effect of lapatinib in canine transitional cell carcinoma cell lines. Vet Comp Oncol 16(4):642-649.

Sebolt-Leopold, J. S.

2000 Development of anticancer drugs targeting the MAP kinase pathway. Oncogene 19(56):6594-9.

Sjodahl, G., et al.

2012 A molecular taxonomy for urothelial carcinoma. Clin Cancer Res 18(12):3377-86.

Solit, D. B., et al.

2006 BRAF mutation predicts sensitivity to MEK inhibition. Nature 439(7074):358-62.

$\mathrm{Su}, \mathrm{H}$, et al.

2019 Hope and challenge: Precision medicine in bladder cancer. Cancer Med 8(4):1806-1816.

Subbiah, V., et al.

2018 Dabrafenib and Trametinib Treatment in Patients With Locally Advanced or Metastatic BRAF V600Mutant Anaplastic Thyroid Cancer. J Clin Oncol 36(1):7-13.

Takeuchi, A., et al.

2012 Sunitinib enhances antitumor effects against chemotherapy-resistant bladder cancer through suppression of ERK1/2 phosphorylation. Int J Oncol 40(5):1691-6.

Usui, T., M. Elbadawy, and K. Sasaki

2018a Session 6: Advances in Anticancer Therapy. Journal of Veterinary Pharmacology and Therapeutics 41(S1):28-30.

Usui, T., et al.

2016 Establishment of a Novel Model for Anticancer Drug Resistance in Three-Dimensional Primary Culture of Tumor Microenvironment. Stem Cells Int 2016:7053872.

Usui, T., et al.

2017 Establishment of a dog primary prostate cancer organoid using the urine cancer stem cells. Cancer Sci 108(12):2383-2392.

Usui, T., et al.

2018b Hedgehog Signals Mediate Anti-Cancer Drug Resistance in Three-Dimensional Primary Colorectal Cancer Organoid Culture. Int J Mol Sci 19(4).

Volkmer, J. P., et al. 
2012 Three differentiation states risk-stratify bladder cancer into distinct subtypes. Proc Natl Acad Sci U S A 109(6):2078-83.

\section{Supporting information}

Additional supporting information may be found online in the Supporting Information section at the end of this article.

\section{Figure Legends}

Fig. 1. Effects of trametinib on cell viability and activation of cell signaling in dog bladder cancer (BC) organoids. Experimental schema of analysis of BC organoids (A). After BC organoids were generated from urine samples of $\mathrm{BC}$ diseased dogs, they were used for the following experiments. Protein expression and activated level of EGF-related signals in BC organoids (B). Expression level of phosphorylation of EGFR, total EGFR, phosphorylation of ERK, and total ERK was compared between normal bladder epithelial cells (NB) and several strains of BC organoids (Or1-5) as determined by Western blotting $(\mathrm{n}=3)$. Equal loading amounts of protein was confirmed by total VCP antibody. Histological analysis of BC organoids (C). Representative images of hematoxylin and eosin (H\&E) staining of each BC organoid were shown. Scale bar: $100 \mu \mathrm{m}$. Representative phase-contrast images (scale bar: $500 \mu \mathrm{m}$ ) of the sensitivity of BC organoids to different concentrations of trametinib for $72 \mathrm{~h}$ using Prestoblue cell viability assay ( $\mathrm{D}, \mathrm{n}=3$ 6) and its quantification (E). 100\% represents cell viability of each control. Results were expressed as mean \pm SEM. Effects of trametinib on activation of EGFR and ERK in BC organoids. After BC organoids were seeded into Matrigel, they were treated with trametinib for 3, 6, 12 and 24 hours. Expression level of phosphorylation of EGFR and ERK was determined by Western blotting (F, G, n=4-5). Equal protein loading was confirmed using total actin antibody. Results were expressed as mean \pm S.E.M. ${ }^{*} P<0.05$ vs. control. Effects of trametinib on expression of downstream signal of ERK in BC organoids. Expression of c-Myc, ETS transcription factor, ETS Like-1 protein (ELK1), Salt-Inducible Kinase 1 (SIK1), and phospholipase A2 group 4A(PLA2G4A) mRNA was determined by quantitative real-time PCR $(\mathrm{H})$. Expression level of each gene was quantified based on the ration of expression level to GAPDH and shown as fold increase relative to control $(\mathrm{n}=4)$. Results were expressed as mean + - S.E.M. ${ }^{*} P<0.05$ vs. control.

Fig. 2. Effects of trametinib on cell cycle arrest and apoptosis in BC organoids. After BC cells were treated with trametinib $(1 \mu \mathrm{M}$ for $24 \mathrm{~h})$, they were stained with propidium iodide (PI). Distribution of cell cycle phases (G0/G1, S, and G2/M) of BC organoid cells was analyzed by flow cytometry (A). The population of $\mathrm{G}_{0} / \mathrm{G}_{1}, \mathrm{~S}$ and $\mathrm{G}_{2} / \mathrm{M}$ phases in $\mathrm{BC}$ organoids was expressed as mean \pm S.E.M $(\mathrm{n}=4)$. ${ }^{*} P<0.05$ vs. control. Effects of trametinib on expression of cell cycle-related genes in BC organoids (B). Expression of cyclin D1, cyclin A2, cyclin E1, CDK4, CDK6, p21, and p16 mRNA was determined by quantitative real-time PCR. Expression level of each gene was quantified based on the ration of expression level to GAPDH and shown as fold increase relative to control $(\mathrm{n}=4)$. Results were expressed as mean +- S.E.M. ${ }^{*} P<0.05$ vs. control. Expression of cyclin D1 in trametinib-treated BC organoids. Representative photomicrographs were shown $(\mathrm{n}=3)$. Scale bar: $50 \mu \mathrm{m}(\mathrm{C})$. Expression level was quantified by counting the cyclin D1-positive cells (D, $\mathrm{n}=3)$. Results were expressed as mean \pm S.E.M. ${ }^{*} P<0.05$ vs. control. Effects of trametinib on apoptosis in $\mathrm{BC}$ organoids $(\mathrm{E})$. After $\mathrm{BC}$ cells were treated with trametinib $(1 \mu \mathrm{M}$ for $72 \mathrm{~h})$, they were stained with terminal deoxynucleotidyl transferase dUTP nick end labeling (TUNEL). DNase-treated organoid cells were used as the positive control $(\mathrm{n}=3)$. The green fluorescence area indicates apoptotic-positive organoids cells and the blue DAPI staining shows intact DNA. Scale bar: $50 \mu \mathrm{m}$. Quantification of apoptosis in trametinibtreated and non-treated cells was analyzed by Image J software $(\mathrm{F}, \mathrm{n}=3)$. Results were expressed as mean \pm S.E.M. ${ }^{*} P<0.05$ vs. control.

Fig. 3. Effects of trametinib on stemness of BC organoids. Expression levels of CD44 and YAP1 mRNA in trametinib-treated $(1 \mu \mathrm{M}, 24 \mathrm{~h}) \mathrm{BC}$ organoids were determined by quantitative real-time PCR (A). Expression level of each gene was quantified based on the ration of expression level to GAPDH and shown as fold increase relative to control $(\mathrm{n}=4)$. Results were expressed as mean \pm S.E.M. ${ }^{*} P<0.05$ vs. control. Expression of CD44 and YAP1 protein in trametinib-treated $(1 \mu \mathrm{M}, 24 \mathrm{~h}) \mathrm{BC}$ organoids. Representative 
photomicrographs were shown $(\mathrm{B}, \mathrm{n}=3)$. Scale bar: $50 \mu \mathrm{m}$. Combinational effects of YAP inhibitor and trametinib on cell viability of $\mathrm{BC}$ organoids $(\mathrm{C})$. Representative phase-contrast images of the trametinib (1 $\mu \mathrm{M})$ alone, in combination with YAP1 inhibitor, verteporfin (VP) $(0.3 \mu \mathrm{M})$, or VP $(0.3 \mu \mathrm{M})$ alone for $72 \mathrm{~h}$ on BC organoids. Scale bar: $500 \mu \mathrm{m}$. The enlarged images were shown below for each image. Cell viability was assessed using Prestoblue kit and $100 \%$ represents the cell viability of each control $(D, n=6)$. Results were expressed as mean \pm S.E.M. ${ }^{*} P<0.05$ vs. control, $\# P<0.05$ vs. trametinib. Apoptosis level was assessed by TUNEL staining in each BC organoid (E). Scale bar: $50 \mu \mathrm{m}$. Quantification of apoptosis in each BC organoid was analyzed by ImageJ software $(\mathrm{F}, \mathrm{n}=4)$. Results were expressed as mean \pm S.E.M. $* P<0.05$ vs. control, $\# P<0.05$ vs. trametinib.

Fig. 4. Effects of trametinib on cell transformation of $\mathrm{BC}$ organoids. Representative bright-field images (A) and $\mathrm{H} \& \mathrm{E}$ staining (B) of the transformed $\mathrm{BC}$ organoids compared with control ones after trametinib $(1 \mu \mathrm{M})$ treatment for $72 \mathrm{~h}$. Scale bar: $500 \mu \mathrm{m}$. The enlarged images were shown below for each image. The ratio of basal-like and luminal-like organoids was quantified by using ImageJ software $(C, n=4)$. Effects of trametinib on expression of basal and luminal marker genes in BC organoids (D). mRNA expression of basal cell markers, CK5 and DSG3 and luminal cell ones, ERBB2 and GATA3 was determined by quantitative realtime PCR. Expression level of each gene was quantified based on the ration of expression level to GAPDH $(\mathrm{n}=4)$. Results were expressed as mean \pm S.E.M. ${ }^{*} P<0.05$ vs. control. Expression of CK5 in trametinibtreated BC organoids. Representative photomicrographs were shown (E). Scale bar: $50 \mu \mathrm{m}$. Expression level was quantified by counting the CK5-positive cells $(\mathrm{F}, \mathrm{n}=3)$. Results were expressed as mean \pm S.E.M. ${ }^{*} P<$ 0.05 vs. control.

Fig. 5. Effects of trametinib on tumor growth against xenografted $\mathrm{BC}$ organoids in immunodeficient mice. Experimental schema of in vivo experiment (A). After BC organoid cells were subcutaneously injected into the back of SCID mice and tumors formed, trametinib and the vehicle was administered to the mice at the concentration of $1 \mathrm{mg} \mathrm{kg}^{-1}$ for 4 weeks. Thereafter, the tumor volume was measured by clipper every week for 6 weeks $(\mathrm{B}, \mathrm{n}=6)$. Tumor tissues of each group was isolated and used for analysis. Comparison of tumor growth curve (B), size (C), and weight (D) between trametinib- or vehicle- administered mice. Results were expressed as mean +- S.E.M. ${ }^{*} P<0.05$ vs. vehicle. Representative H\&E staining images of the tumor tissue sections from trametinib- or vehicle- administered mice (E). Scale bar: $200 \mu \mathrm{m}$. Confocal microscopy fluorescence images of apoptosis as determined by TUNEL staining of frozen sections of tumor tissues from trametinib-treated or vehicle- administered mice $(\mathrm{F}, \mathrm{n}=3)$. Sections treated with DNase I were used as a positive control. Scale bar: $50 \mu \mathrm{m}$. Expression level of CK5 in sections from dissected tumors of trametinibor vehicle-administered mice as determined by confocal microscopy $(\mathrm{G}$, scale bar: $50 \mu \mathrm{m})$. Expression level was quantified by counting the CK5-positive cells $(\mathrm{H}, \mathrm{n}=3)$. Results were expressed as mean \pm S.E.M. ${ }^{*} P<$ 0.05 vs. vehicle.

Fig. 6. Effects of long-term trametinib administration to mice on drug sensitivity of xenograft-derived organoids. Experimental schema of generation of xenograft-derived organoids from trametinib administered and vehicle-administered mice and the analysis (A). Bright field (B) and H\&E staining (C) images of the vehicle (XDO-C) and trametinib (XDO-T) administered mice-derived tumor organoids. Scale bar: $500 \mu \mathrm{m}$. Sensitivity to trametinib (D, $n=3-6)$ and carboplatin $(\mathrm{E}, \mathrm{n}=3-6)$ was analyzed by Prestoblue kit. Results were expressed as mean \pm S.E.M. $100 \%$ represents the cell viability of each control. Expression of a carboplatin sensitivity-related gene, MSH2 mRNA in each organoid was determined by quantitative real-time PCR. Expression level of gene was quantified based on the ration of expression level to GAPDH (F, $\mathrm{n}=4)$. Results were expressed as mean \pm S.E.M. ${ }^{*} P<0.05$ vs. vehicle. Expression level of MSH2 in xenografted tumorderived organoids and original tissues $(\mathrm{G}, \mathrm{n}=3)$ from trametinib- or vehicle-administered mice. Scale bar: 50 $\mu \mathrm{m}$. Schematic summary of the present study $(\mathrm{H})$. Trametinib promoted cell cycle arrest, inhibited stemness, transformed the phenotype of $\mathrm{BC}$ organoids, which may lead to the inhibitory effects of tumorigenesis in vivo. Besides, long-term trametinib administration enhanced the sensitivity to carboplatin through upregulation of MSH2 gene in the tumor tissues.

\section{Hosted file}


Dog bladder cancer trametinib paper Figure. 10.16..20-2.pdf available at https://authorea. com/users/368546/articles/487612-anti-tumor-effect-of-trametinib-in-bladder-cancerorganoid-and-the-underlying-mechanism

\section{Hosted file}

BJP Table.10.12..20.xlsx available at https://authorea.com/users/368546/articles/487612-antitumor-effect-of-trametinib-in-bladder-cancer-organoid-and-the-underlying-mechanism 